\title{
Measurements of short wavelength VLF bursts in the auroral ionosphere: A case for electromagnetic mode conversion?
}

\author{
G. T. Delory, R.E. Ergun, E.M. Klementis, C.W Carlson, and J.P. McFadden \\ Space Sciences Laboratory, University of California at Berkeley
}

\begin{abstract}
Intense, localized bursts of VLF emissions were measured on the University of California at Berkeley Alaska '93 auroral sounding rocket. A wavelength determination for these waves was obtained using cross-spectral analysis on high time resolution electric field measurements from dipole antennas and an onboard burst memory system. The observation of an antenna null in the cross-spectral response reveals very short wavelength emissions with wavelengths less than 5.5 meters. The applicability of a collapse theory for these emissions is put in question since these examples are not well correlated with electron density depletions. In addition, these waves do not appear to be associated with a particular type of electron distribution which may have been responsible for their generation. The presence of electromagnetic modes in the VLF frequency range in the vicinity of many of these electrostatic bursts, along with continual electron density turbulence of $1-5 \%$, may provide evidence for a linear mode coupling mechanism in which electrostatic VLF waves are produced by the scattering of incident electromagnetic whistlers from electron density irregularities.
\end{abstract}

\section{Introduction}

Observations of intense, localized packets of Very Low Frequency (VLF) bursts have been made by numerous sounding rockets in the auroral ionosphere [Vago et al., 1992; Ergun et al., 1993]. In general, VLF waves are either electrostatic emissions propagating near the cold plasma resonance cone from the local lower hybrid frequency $\left(f_{\mathrm{lh}}\right)$ up to the electron plasma or cyclotron frequencies, or electromagnetic emissions over the same frequency range with wavelengths on the order of kilometers. Under auroral conditions these waves appear as broadband noise in the electric field from $f_{\mathrm{lh}} \sim 2-7 \mathrm{kHz}$ extending up to $500 \mathrm{kHz}-1 \mathrm{MHz}$. The observation of intense bursts of VLF waves near $f_{\mathrm{lh}}$ with a localized time series signature and short wavelengths of less than 20 meters has stimulated a renewed interest in these emissions as candidates for non-linear wave collapse and as a possible mechanism for the phenomenon of ion heating in the auroral ionosphere. Vago et al. [1992] and Labelle et al. [1986] have measured Transversely Accelerated Ions (TAI's) in conjunction with "spikelets," high-amplitude lower hybrid waves which have coupled to the ambient electron plasma via the ponderomotive force and have collapsed into shorter wavelengths through a modulational instability, creating an electron density depletion in the process [Chang, 1993]. Electron density depletions associated with waves in the VLF regime have been reported on both the FREJA spacecraft [Pécseli et al., 1995] as well as on the TOPAZ III auroral sounding rocket in the work by Vago et al.

Short wavelength VLF waves near the lower hybrid frequency are of great interest because of the possibility that they are responsible for ion heating, creating large fluxes of upwelling ions which may make a significant contribution to the magnetospheric plasma population. VLF waves are an attractive candidate for ion heating because these emissions may gain energy from precipitating auroral electrons via their small parallel wave numbers $\left(k_{\|}\right)$and then energize the cold ion population via their much larger perpendicular wave numbers $\left(k_{\perp}>k_{\|}\right)$and electric fields. However even the shortest wavelength VLF emissions that can be produced easily by resonant growth from typical inverted-V electron populations have perpendicular wavelengths on the order of 100's of meters; thus the collapse model has been proposed as a mechanism to bridge the gap between the wavelengths of VLF waves generated by the auroral beam and the wavelengths necessary for these waves to heat ionospheric ions, which typically need wavelengths of less than a few meters for efficient energization.

In this letter we show measurements of extremely short wavelength VLF emissions obtained from a University of California at Berkeley auroral sounding rocket flown in 1993. The short wavelength character of these emissions, less than $5.5 \mathrm{~m}$ near $f_{\mathrm{lh}}$, is obtained using a null in the coherency spectrum coupled with a characteristic phase angle shift of the emissions in cross spectral analysis of the electric field time series data. Simultaneous measurements of the electron density fluctuations by a separate Langmuir probe reveal no consistent electron density depletions associated with these events. However, the electron density measurements do show turbulent fluctuations on the order of $1-5 \%$ throughout the flight, as well as the presence of circularly polarized, presumably electromagnetic emissions in the VLF range in close proximity to the intense VLF bursts. Linear mode coupling of incident electromagnetic modes scattering off of electron density perturbations into electrostatic VLF waves is one possibility that has been investigated numerically by Bell \& Ngo [1990], and is a model that appears least contradictory to our observations.

\section{Experimental Observations and Analysis}

The UCB Alaska '93 auroral sounding rocket was launched April 2, 1993 from the Poker Flat rocket range into an evening auroral substorm. The payload consisted of a set of voltage probes which obtained 3-D AC and DC electric field measurements as well as ion and electron electrostatic analyzers for measuring particle distributions. The electric field data presented here is from the onboard High Speed Burst Memory (HSBM), which used a trigger threshold criteria to select high amplitude bursts of electric fields in the VLF frequency range for storage in a high time resolution memory buffer. The HSBM provided $\sim 2 \%$ coverage of VLF bursts, yielding 20 millisecond long snapshots of time series electric field data out of every second of real time sampled at $100 \mathrm{kHz}$ by analog-to-digital converters.

Figure 1 shows two of the rocket probes used for the wavelength analysis. The vehicle is spin-aligned with the major axis along the geomagnetic field with a spin period of $\sim 1.28$ seconds; during the measurements described here the vehicle velocity perpendicular to the geomagnetic field $\mathbf{B}$ was $\sim 1.3 \mathrm{~km} / \mathrm{sec}$ and had an anti-parallel component of $\sim 1.5 \mathrm{~km} / \mathrm{sec}$. Electrostatic VLF emissions near the lower hybrid frequency will propagate with group velocities nearly along $\mathbf{B}$ and phase velocities and electric fields 


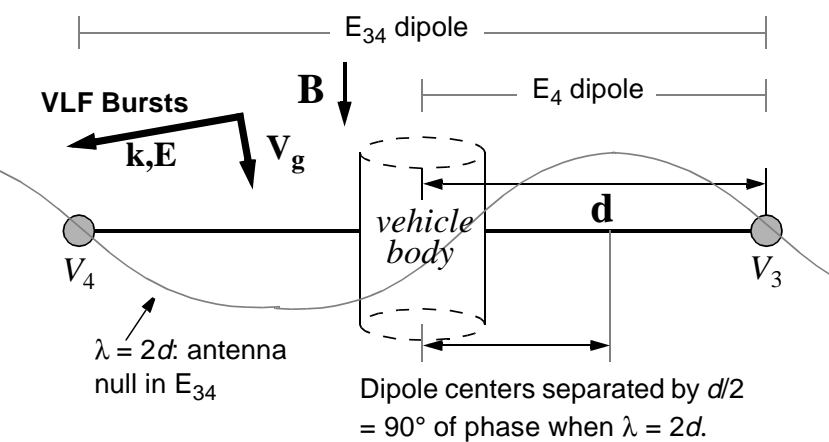

Figure 1. A cross section view of the electric field probes used for the cross-spectral analysis.

oriented nearly perpendicular to $\mathbf{B}$. Optimal measurements for the VLF burst wavelength determination are obtained from the spherical voltage probes $V_{3}$ and $V_{4}$ which provide the electric field perpendicular to $\mathbf{B}$ using $\mathrm{E}_{34}=\left(V_{3}-V_{4}\right) / 2 d$. This yields the component of the electric field along probes $V_{3}$ to $V_{4}$. A second signal, obtained by subtracting the $V_{4}$ potential from the potential of the vehicle skin, yields $\mathrm{E}_{4}=\left(\mathrm{V}_{\text {payload }}-V_{4}\right) / d$. This measurement also represents the electric field along the $V_{3}-V_{4}$ direction, however it is obtained using a shorter dipole that is displaced a distance $d / 2$ from the dipole used for the $\mathrm{E}_{34}$ measurement. Cross spectral analysis can then be applied to the $E_{34}$ and $E_{4}$ signals to measure the coherence and phase delay between the two dipole measurements.

The technique of cross spectral analysis has been reviewed by Labelle \& Kintner [1989] and applied experimentally on the TOPAZ III sounding rocket data in the work by Vago et al. Fast Fourier transforms (FFT's) of the electric field measurements $\mathrm{E}_{34}$ and $\mathrm{E}_{4}$ can be obtained yielding complex Fourier spectra $S_{34}(\omega)$ and $S_{4}(\omega)$. The normalized cross spectra of these two signals is then formed using

$$
C(\omega)=\frac{\left\langle S_{34}^{*}(\omega) S_{4}(\omega)\right\rangle}{\left\langle S_{34}^{2}(\omega)\right\rangle^{1 / 2}\left\langle S_{4}^{2}(\omega)\right\rangle^{1 / 2}}=\gamma(\omega) e^{i \Theta(\omega)}
$$

The asterisk $(*)$ denotes the complex conjugate of the FFT of each signal, and the delimiters \langle\rangle denote a segmented or sliding average of the spectra over a specified record length of data. The complex quantity $C(\omega)$ can be re-expressed in terms of a magnitude and a phase, where $\gamma^{2}(\omega)$ is referred to as the coherency function, while $\Theta(\omega)$ is the phase difference between the two input signals. For signals with a definite phase relationship between successive wavefronts, the coherency will be close to unity and a reliable phase delay between the signals can be determined. For random, uncorrelated noise measured from each dipole antenna, the coherence will be poor and the phase measurement will be indeterminate. For electrostatic waves the phase angle $\Theta(\omega)=\mathbf{k} \cdot \mathbf{d} / 2$, yielding the wave number spectrum assuming that the separation between the $\mathrm{E}_{34}$ and $\mathrm{E}_{4}$ dipoles $=d / 2$ and the angle between $\mathbf{k}$ and d are known. For the Alaska '93 payload, $d=2.75$ meters.

Figure 2 shows electron density perturbations, the root-mean -square (RMS) electric field from the $V_{3}-V_{4}$ probes ("E34") averaged over $1-16 \mathrm{kHz}$, and an HSBM capture showing the actual waveform of one of the VLF bursts at $\mathrm{T}=410.09$ seconds into the flight. Note that bursts of VLF wave power are recorded during both enhancements and depletions of electron density which are

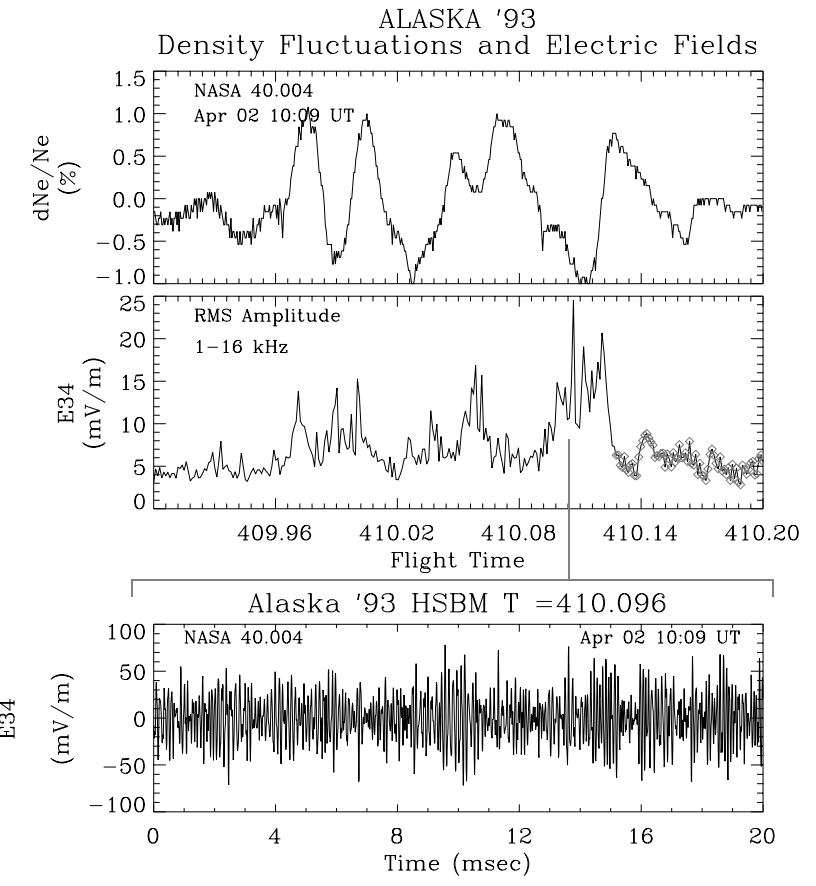

Figure 2. Electron density fluctuations, RMS electric field, and an HSBM capture of intense VLF wave power near $\mathrm{T}=410.09 \mathrm{sec}$. never more than fluctuations of $1 \%$. Figure 3 shows the cross spectrum and FFT of the HSBM event shown in Figure 2. The top plot in Figure 3 shows the coherency, which is close to $\sim 1$ over a broad frequency range between 2.5 and $40 \mathrm{kHz}$. Below $2.5 \mathrm{kHz}$ and above $40 \mathrm{kHz}$, low amplitude, random uncorrelated noise seems to dominate the signal as shown by the low coherency at these frequencies. The phase angle plot shows a smoothly increasing phase delay between the $\mathrm{E}_{4}$ and $\mathrm{E}_{34}$ signals with decreasing frequencies, going from near $0^{\circ}$ at $40 \mathrm{kHz}$ to $>80^{\circ}$ just above $5 \mathrm{kHz}$, and the FFT shows a peak in the spectra above the lower hybrid frequency $(4-5 \mathrm{kHz})$ with a maximum near $7 \mathrm{kHz}$. For a

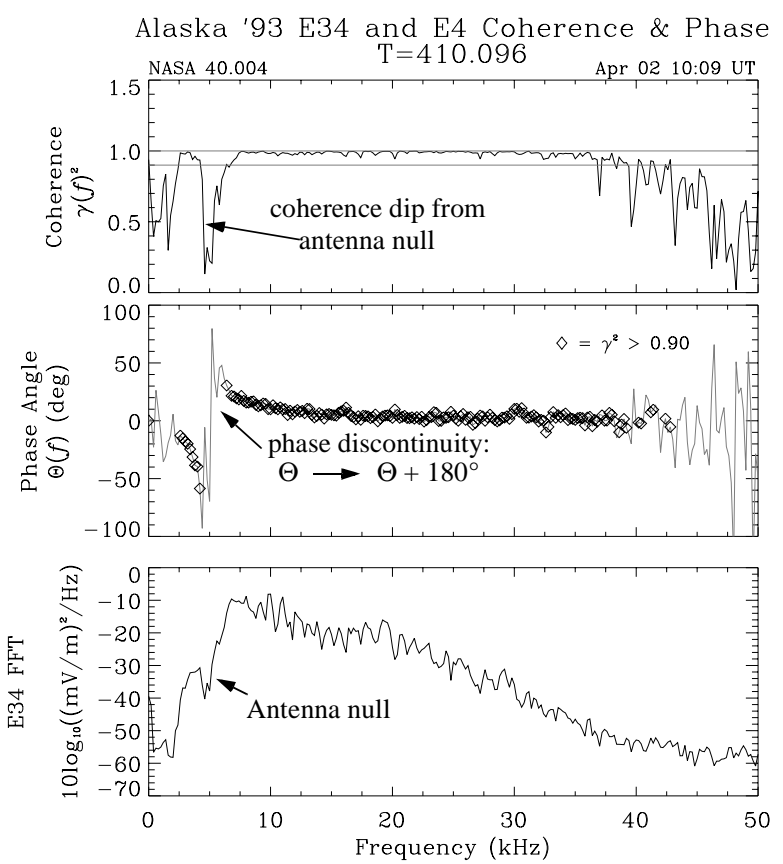

Figure 3. Cross spectral analysis of a VLF burst from HSBM data at $\mathrm{T}=410.096$ seconds into the Alaska '93 flight. 
comparison, spectra during low-amplitude $(<10 \mathrm{mV} / \mathrm{m})$ background VLF hiss a few seconds later has a steep cut-off near $4.5 \mathrm{kHz}$ ( $>40 \mathrm{~dB}$ drop in power in less than $1 \mathrm{kHz}$ ), and a phase angle of $\sim 0^{\circ}$ from $f_{\mathrm{lh}}$ to $\sim 30 \mathrm{kHz}$ where the power drops off.

An initially puzzling yet consistent feature of the cross spectra of the $\mathrm{T}=410.09 \mathrm{sec}$ HSBM event as well as many others during the $\mathrm{T}=400$ to 500 second interval is the observation of a pronounced dip in the coherency spectrum between $3-5 \mathrm{kHz}$. Within the same range of frequencies there is a sudden discontinuity in the phase angle measurement, as it jumps from approximately $90^{\circ}$ to $-90^{\circ}$ and then continues to smoothly rise to $\sim 0^{\circ}$ before the coherency of the signal is lost. Accompanying both the dip in the coherence spectra and the discontinuity in the phase angle measurement is a small dip in the power spectra shown in the FFT of the $E_{34}$ signal at the same frequency. None of these features appeared in the cross-spectra of the low-amplitude background VLF. The dip in the power spectrum of the $E_{34}$ signal for the bursty VLF waves provides the first clue for the origin of this particular feature of the spectrum. The $\mathrm{E}_{34}$ signal will possess a null in the power spectrum whenever the wavelength of the emission is equal to the probe-to-probe separation of the $\mathrm{E}_{34}$ dipole $(\lambda=2 d)$. Additionally, it can be shown [Delory, 1996] that the antenna null for this critical wavelength is accompanied by a sudden phase shift of $180^{\circ}$ of the measured signal. Assuming from the phase angle measurements that the wavelengths are slowly decreasing (phase angle increasing) with lower frequencies, there will be a frequency where the wavelength of the emission is equal to the $\mathrm{E}_{34}$ dipole length but still longer than the $\mathrm{E}_{4}$ dipole length, thus only the $\mathrm{E}_{34}$ signal sees a power loss coupled with a sudden $180^{\circ}$ phase shift while the $\mathrm{E}_{4}$ signal does not. The cross-spectral phase angle routine plots increasing phase from $0^{\circ}$ to $180^{\circ}$ and $-180^{\circ}$ back to $0^{\circ}$ depending on the sign of $\mathbf{k} \cdot \mathbf{d}$, with a phase wrap occurring between $180^{\circ}$ and $-180^{\circ}$. Simulations [Delory, 1996] using a model spectral profile that increased the phase angles with decreasing frequencies demonstrate identical effects as seen in the data in Figure 3. The jump in phase between the $E_{34}$ and $E_{4}$ signals always happens near $90^{\circ}$ because these dipoles are separated by a distance $d / 2$. When the wavelength of the observed emission is equal to the $\mathrm{E}_{34}$ dipole length of $2 d$, a separation distance of $d / 2$ between the dipoles represents $1 / 4$ of a wavelength which translates to $90^{\circ}$ in the relative phase measurement. The instantaneous addition of $180^{\circ}$ of phase when $\lambda=2 d$ then carries the phase measurement from $90^{\circ}$ to $180^{\circ}$, then the phase wraps from $180^{\circ}$ to $-180^{\circ}$, and continues to $-90^{\circ}$. Wavelengths shorter then the $E_{34}$ probe separation then bring the phase measurement from $-90^{\circ}$ toward $0^{\circ}$.

Thus a dip in the wave coherence for these VLF wave bursts, coupled with a phase angle discontinuity and a corresponding bite-out in the FFT spectra of the longer baseline electric field signal $E_{34}$, represent the presence of short wavelength emissions with wavelengths comparable to or less than $5.5 \mathrm{~m}$. A summary plot of the wave coherence, obtained from a compilation of discrete burst memory captures, is shown in Figure 4 along with the electron spectra recorded from $\mathrm{T}=390$ to 510 seconds into the flight. The coherency spectrogram in Figure 4(b) shows a horizontal band where the coherency drops, extending from $\sim 400$ to 480 seconds between 3-5 kHz. This dip in the coherency spectrum on the summary plot is the same phenomena as the single dip near $5 \mathrm{kHz}$ shown in the detailed cross-spectrum of the single VLF burst event at $\mathrm{T}=410.09$ seconds shown in Figure 3. The Alaska '93 flight recorded approximately $\sim 80$ seconds of VLF burst activity that

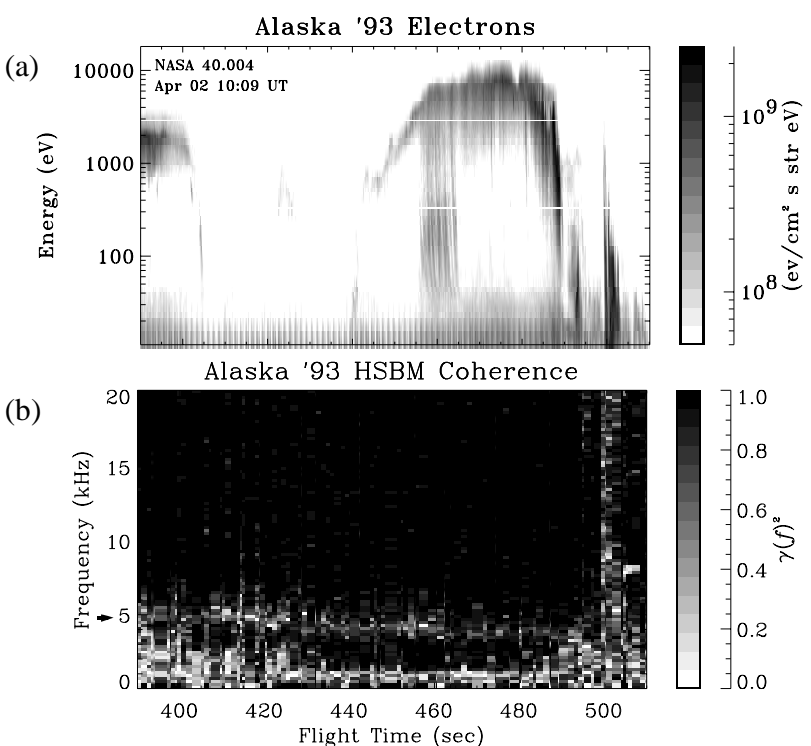

Figure 4. (a) Electron spectrogram and (b) VLF wave coherence spectra. The horizontal band running across the bottom of the coherence plot ranging from $3-5 \mathrm{kHz}$ in frequency indicates an antenna null in the $\mathrm{E}_{34}$ probe due to VLF wavelengths of $<5.5 \mathrm{~m}$.

possessed wavelengths less than or equal to 5.5 meters.

The period from $\mathrm{T}=400$ to 500 seconds in the flight is the only interval with a clear antenna null signature in the coherency spectra. There is little correlation between the short-wavelength VLF bursts in this time interval and electron activity as can be seen from Figure 4(a). As described in the introduction, continual electron density turbulence was recorded, with $1-5 \%$ variations occurring over time scales ranging from 20 to 100 msecs. A direct correlation between electron density depletions and the intense VLF bursts was not evident in the data (see Figure 2), which was complicated by the presence of general background density turbulence as well as several payload-spin dependent features. While some of the VLF bursts in the $\mathrm{T}=400$ to 500 second interval were associated with density depletions of a few percent, there were an equal number occurring during a density enhancement or with no density change at all. Immediately surrounding many of the short-wavelength, electrostatic bursts of VLF waves were circularly polarized VLF emissions with a near zero phase angle measurement and extremely high coherence $(\sim 0.99-1.0)$. These emissions were almost certainly electromagnetic, although there were no AC magnetic field measurements in the VLF range to absolutely confirm this assertion. Initial data from the FAST satellite, however, has shown both electromagnetic as well as electrostatic VLF bursts in the auroral region using simultaneous electric and magnetic field measurements. In addition to the electromagnetic VLF waves there is the possibility of the presence of Hydrogen Bernstein modes in the electrostatic emissions, given that a typical $\mathrm{H}^{+}$ion Larmor radius was $\sim 1-2 \mathrm{~m}$ assuming a $0.1-0.3 \mathrm{eV}$ temperature, which is on the same order as the measured wavelengths of $5.5 \mathrm{~m}$. However these emissions usually manifest themselves with multiple harmonics, and the coherence dip/phase angle discontinuity was a clearly singular feature of the spectra for all of the examples of the short-wavelength VLF bursts.

\section{Interpretation and Theory}

The observation of continual electron density turbulence throughout the flight may suggest a viable explanation for the cre- 
ation of these short-wavelength VLF emissions. Scattering of electromagnetic waves off of the measured density perturbations could be responsible for the generation of much shorter wavelength electrostatic modes with wavelengths on the same scale as the scale size of the density turbulence. Bell \& Ngo [1990] showed that electron density changes as small as a few percent could effectively linearly couple electromagnetic whistlers into electrostatic and electromagnetic modes in auroral plasma conditions. Under this model, the amplitude of the resulting electrostatic VLF bursts could be as high as $30 \mathrm{~dB}$ above the input amplitude of the electromagnetic whistler, assuming that the density striations are field-aligned and the whistlers scatter with a grazing incidence. Bamber et al. [1995] have measured this process experimentally in a laboratory using plasma conditions scaled to auroral parameters. The electromagnetic VLF emissions were ubiquitous throughout the flight and many were in very close proximity (within 5-10 msec) to some of the short-wavelength, electrostatic VLF bursts which had a linear rather than a circular polarization. Many of these examples were accompanied by sudden changes in the electron density of $\sim 1-5 \%$. As is evident from Figure 4 (a) there were no good correlations between the short-wavelength VLF wave bursts and the energetic electron activity, nor were there consistent electron density depletions during many of these events. The simultaneous features that were obvious in the data consisted of the short-wavelength VLF bursts, electron density turbulence, and much longer wavelength, presumably electromagnetic VLF waves. The electromagnetic VLF component is capable of traveling far from its source region and thus no particular correlation with the electrons is expected. This model relies on some pre-existing mechanism for producing electron density turbulence which enables the scattering of the incoming electromagnetic VLF waves into electrostatic emissions.

There are several ambiguities in the application of the wave collapse models to our observations. The maximum density depletions measured on Alaska ' 93 are $~ 10 \%$, which is a rare event, in contrast to the $20-80 \%$ depletions reported in the TOPAZ sounding rocket results from Vago et al. Seyler [1994] has shown that density depletions of even a few percent are sufficient to facilitate VLF wave collapse into solitary structures with short wavelengths, however the results from the Alaska ' 93 measurements indicate a wide variety of density profiles associated with the discrete VLF bursts that range from depletions to enhancements. Even when waves in the VLF regime are clearly associated with electron density depletions, there is still some controversy in the interpretation of the measurements; Pécseli et al. have shown examples of lower hybrid wave cavities recorded on the FREJA mission which are claimed to be inconsistent with the time and length scales predicted by a collapse scenario. Recently Robinson et al. [1996] have stated that wave collapse in the lower hybrid regime may occur during both electron density depletions and enhancements for waves with opposite senses of elliptical polarization. Our data is not inconsistent with such a model, however a more detailed wave polarization analysis is necessary and will be the subject of a future work. For our results, a wave collapse scenario is applicable only if (1) the initial electron density depletions are pre-existing before the creation of the VLF bursts, (2) the VLF waves may collapse in the vicinity of electron enhancements as well as depletions of a few percent, and (3) the collapse scenario produces VLF bursts with a coherent dispersion relation, with a definite wave number for each frequency. Such a dispersion relation for the measured VLF bursts is implicit in the observed antenna null effects and in the phase-angle spectra shown in Figure 3.

\section{Conclusion}

Using an antenna null effect in the cross spectral analysis of intense, transient VLF emissions, we have isolated a short-wavelength component of VLF electric field bursts with wavelengths less than 5.5 meters. Of the possibilities considered, the scattering of electromagnetic VLF waves from the continual density turbulence observed throughout the flight is the only mechanism that does not directly contradict our data as a means for producing short-wavelength, electrostatic VLF bursts. We base this conclusion on the simultaneous observations of electron density fluctuations, short-wavelength VLF bursts, and long-wavelength electromagnetic emissions in the VLF range. Other possibilities, namely caviton formation within electron density enhancements as well as depletions, require further examination given our data set. Finally, we do not find that these short wavelength examples of VLF bursts are associated with significant ion heating.

Acknowledgements. The authors thank Dr. Robert Pfaff for useful discussions. This research was supported by NASA grant NAG6-10.

\section{References}

Bamber, J.F., J.E. Maggs and W. Gekelman, Whistler wave interaction with a density striation: A laboratory investigation of an auroral process, J. Geophys. Res., 100, 23795-23810, 1995.

Bell, T.F., and H.D. Ngo, Electrostatic lower hybrid waves excited by electromagnetic whistler mode waves scattering from planar magnetic-field-aligned plasma density irregularities, J. Geophys. Res., 95, 149-172, 1990.

Chang, T., Lower-hybrid collapse, caviton turbulence, and charged particle energization in the topside auroral ionosphere and magnetosphere, Phys. Fluids B 5(7), 2646-2656, 1993.

Delory, G.T., Rocket observations of VLF bursts, electron precipitation, and ion heating in the auroral ionosphere, Ph.D. thesis, 128 pp., University of California at Berkeley, May 1996.

Ergun, R.E., G.T. Delory, E. Klementis, C.W. Carlson, J.P. McFadden, I. Roth, and M. Temerin, VLF wave growth from dispersive bursts of field-aligned electron fluxes, J. Geophys. Res., 98, 3777-3787, 1993.

LaBelle, J., and P.M. Kintner, The measurement of wavelength in space plasmas, Rev. Geophys., 27, 4, 495-518, 1989.

Labelle, J., P.M. Kintner, A.W. Yau, and B.A. Whalen, Large amplitude wave packets observed in the ionosphere in association with transverse ion acceleration, J. Geophys. Res., 91, 7113-7118, 1986.

Pécseli, H.L., K. Iranpour, Ø. Holter, B. Lybekk, and J. Holtet, Lower hybrid wave cavities detected by the FREJA satellite, J. Geophys. Res., 101, 5299-5316, 1996.

Robinson, P.A., A. Melatos, and W. Rozmus, Is there lower hybrid wave collapse at auroral altitudes? J. Geophys. Res., 101, 21545-21552, 1996.

Seyler, C.E., Lower hybrid wave phenomena associated with density depletions, J. Geophys. Res., 99,19513-19525, 1994.

Vago, J. L., P. M. Kintner, S. W. Chesney, R. L. Arnoldy, K. A. Lynch, T. E. Moore and C. J. Pollock, Transverse ion acceleration by localized lower hybrid waves in the topside auroral ionosphere, J. Geophys. Res., 97, 16935-16957, 1992.

C.W. Carlson, G.T. Delory, R.E. Ergun, E.M. Klementis, and J.P. McFadden, Space Sciences Laboratory, University of California at Berkeley, Berkeley, CA 94720-7450 (e-mail: gdelory@ssl.berkeley.edu).

(Received: November 12, 1996; revised: March 11, 1997; accepted: March 18, 1997) 